\title{
A Dynamic System Interpretation of Irreducible Complexity
}

\author{
ABRAHAM BOYARSKY and PAWEL GÓRA* \\ Department of Mathematics and Statistics, Concordia University, 7141 Sherbrooke Street West, Montreal, Que., Canada H4B IR6
}

(Received 24 April 2001)

\begin{abstract}
Behe recently defined the idea of irreducible complexity for biological systems. Using the language of mathematics, we reinterpret his definition from a dynamical systems perspective. Our basic premise is that living organisms behave dynamically in a chaotic way while predictable periodic behavior reflects cessation of function. We consider the dynamics of a functioning system and altered versions of it to draw conclusions about the irreducible complexity of the original system. The dynamics of an organism is described by means of a discrete time transformation $\tau$ on the phase space of the system. The statistical behavior of $\tau$ is studied by means of its Frobenius-Perron operator which, in special cases, can be represented by a matrix. Using these matrices we rewrite our definition of irreducible complexity: $M$ is irreducibly complex if it is primitive but no principal submatrix of $M$ is primitive. The primitivity property implies chaotic behavior, while failure to have the primitivity property reflects periodic behavior. Examples of irreducibly complex dynamical systems are presented. We show that certain dynamical systems which are irreducibly complex have an additional property, namely that other systems arbitrarily close to it behave in a dramatically different way. Such behavior suggests that selective evolution by means of small perturbations may not be a general mechanism for achieving the dynamical behavior of a complex system.
\end{abstract}

Keywords: Dynamical system; Irreducible complexity; Frobenius-Perron operator; Chaotic dynamics

\section{INTRODUCTION}

There exist organisms that consist of a finite number of distinct interdependent components and present complex behavior in the sense that all the components must be in place before it is a meaningfully functional organism. If any component is missing the subsystem does not function meaningfully and hence does not offer any advantage to the larger system as demanded by selective evolution.

In this note we attempt to use the language of non-linear dynamics and chaos theory to give mathematical meaning to the phrase "irreducible complexity". In Section 2 we present a brief overview of chaotic dynamics, in particular the role of the Frobenius-Perron operator and its matrix representation for piecewise linear Markov maps. Behe's notion of irreducible complexity (Behe, 1996) is reinterpreted from the perspective of dynamical systems theory. It follows from our Definition 2 that a system is irreducibly complex if the matrix representation for the associated Frobenius-Perron operator is primitive but such that no principal submatrix has this property. This is tantamount to requiring that the deletion of any part of the state space results in a non-functioning organism. Periodic behavior may result, but the chaos (deterministic randomness) that is the hallmark of a living organism is no longer present. In Section 3 we give a number of examples of such irreducibly complex dynamical systems. In Section 4 we show that certain dynamical systems have the property that, no matter how close other dynamical systems are to it, the dynamical behavior of nearby systems are very different from that of the original system. That is, the complex dynamics of the original system cannot be achieved by arbitrarily close systems.

\section{REVIEW OF CHAOTIC DYNAMICS}

It is now common knowledge that even simple onedimensional maps have the ability to describe very complicated dynamical behavior of biological and mechanical systems (Lasota and Rusck, 1974; May, 1976; Guevera and Glass, 1982; West, 1990; Shinbrot et al., 1992; Liebovitch and Krekora, 2000). Modeling dynamics by a map offers much more variety of behavior

*Corresponding address. Tel.: +1-514-848-3240. Fax: +1-514-848-2831. E-mail: pgora@ vax2.concordia.ca 
than do differential equations whose solutions are greatly restricted by time continuity and cannot exhibit chaotic behavior in low dimensions. Describing dynamical behavior by iterating a map, which can arise as a Poincare section or by direct modeling (Liebovitch and Krekora, 2000), offers many benefits from an analysis perspective. Once a map is determined, the long term statistical behavior is described by a probability density function (pdf), which can be obtained by measurement of the system or by mathematical means using the FrobeniusPerron operator (Boyarsky, 1996) as follows: let $I=$ $[a, b]$ denote the state space of a dynamical system and let $\tau: I \rightarrow I$, describe the dynamics of the system. We assume the system behaves chaotically and that this chaotic behavior is described by a probability density function $f$ associated with the unique (absolutely continuous invariant) measure $\mu$. This is stated mathematically by the following equation:

$$
\int_{A} f \mathrm{~d} x=\int_{\tau^{-1} A} f \mathrm{~d} x
$$

for any (measurable) set $A \subset \mathbb{R}$. The Frobenius-Perron operator, $P_{f} f$, acts on the space of integrable functions and is defined by

$$
\int_{\tau^{-1}(A)} f \mathrm{~d} x=\int_{A} P_{\tau} f \mathrm{~d} x
$$

The operator $P_{\tau}$ transforms probability density functions into probability density functions under the transformation $\tau$. If $\tau$ is Markov with respect to a partition $\mathscr{P}$ of I that is, it maps elements of $\mathscr{P}$ onto unions of elements of $\mathscr{P}$, then $P_{\tau}$ has a matrix representation, $M_{\tau}$, where the $i$ th row consists of (contiguous) non-zero elements only where the image of the $i$ th interval intersects the $j$ th interval and the entry is 1/slope of $\tau$ on the $i$ th interval (see Boyarsky, 1997, Ch. 9) for more details. The fixed points of $P_{\tau}$ are the observed probability density functions (pdf). If $\tau$ is a Markov map, then the (normalized) left eigenvectors of the matrix $M_{\tau}$, viewed as functions on $I$, represent the fixed points.

\section{IRREDUCIBLE COMPLEXITY}

By irreducible complexity Behe (Behe, 1996) means that the organism consists of a finite (possibly large) number of independent components and that these components are coupled in some way such that, if any component is removed, the reduced system no longer functions meaningfully. Most prominent among the biological systems that appear to have this property is the much studied bacterial flagellar motor (Schuster and Kahn, 1994; Caplan and Kara-Ivanov, 1993).

DEFInITION 1 A system is called "irreducibly complex" if no simpler form of it functions meaningfully (Behe, 1996).
We abstract from Definition 1 the following: the term "complexity" refers to the number of components in the system, and "irreducible" to the fact that the function of any reduced system is dramatically different from the original system. In this note we propose a dynamical interpretation of Definition 1: we interpret "complexity" to refer, not to the number of components of the original system, but rather to the dynamic complexity of the system. By "irreducibility" we mean that the state space of the system cannot be reduced without causing the complex dynamical behavior to change dramatically, such as, for example, collapsing from chaotic (seemingly random) behavior to completely deterministic periodic behavior.

Before we present a more precise definition, we need the notion of the state of the system. By the state of a system we mean a point on an interval (or an $N$ dimensional subset of $\mathbb{R}^{N}$ ) whose value captures the dynamics of all the variables that are relevant in describing the system. A system consisting of $N$ components can be mathematically described as a point in $N$-dimensional space $\mathbb{R}^{N}$, where each coordinate gives a number describing the state of the corresponding components. Since the parts are assumed to be interconnected, the coordinate points do not attain every possible position in $\mathbb{R}^{N}$, but rather move along some lower dimensional subset of $\mathbb{R}^{N}$, say, of dimension $K<N$, which is referred to as the phase manifold. Such a manifold can be (at least locally) parameterized by points of a $K$-dimensional space $\mathbb{R}^{K}$. If the movement of the organism is bounded, and any physical system will be, a parameterization using the $K$-dimensional cube $[0,1]^{K}$ can be chosen. For convenience we assume that the phase manifold can be mapped onto an interval $I$. The dynamics of the entire system is now reflected in the dynamics of the one-dimensional map $\tau$ on the state space $\mathrm{I}$.

We say a dynamical system defined by the map $\tau$ is complex if its long-term behavior is described by a probability density function (pdf), that is, it behaves chaotically and it is "irreducible" if, by removing certain parts of the state space, the dynamics of the reduced system collapses to (completely predictable) periodic behavior. Irreducible in this sense implies irreducible complexity in the sense of Definition 1. More precisely, we have Definition 2.

DEFINITION 2 Let $I$ denote the state space of a onedimensional dynamical system $\tau: I \rightarrow I$ that possesses a pdf of $f$. We say the dynamical system described by $\tau$ and $f$ is "irreducibly complex" if there exists a number $\in \geq 0$, less than the measure of $I$, such that if any interval $J$ of length $\in$ is removed, then the dynamics of the reduced system $\tau / I-J$ collapses to periodic behavior.

Any system that is irreducibly complex in the sense of Definition 2 is irreducibly complex in the sense of Definition 1. What is common to the two definitions is the idea that a "reduction" in the original system causes failure of operation. In Definition 1, the reduction is due to 


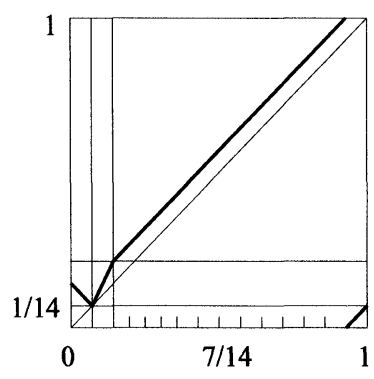

FIGURE 1

an actual removal of a physical component of the system. In Definition 2 the reduction is a set of states in the phase space of the system. Since a physical reduction (such as the removal of a component) of a mechanism may cause a reduction in the attainable states of the system the two definitions reflect the same event in different ways. For example, consider an organism with $n$ components producing a velocity that is its measurable state. Now, paralysis of a component (a muscle, say) results in a simpler form of the original system (as used in Definition 1) and at the same time results in a reduced set of attainable velocities (as used in Definition 2).

In practice one encounters data in the form of a phase plot, where the state $x(n+1)$ is plotted against $x(n)$ The dynamical system $\tau$ is a map whose graph is inside the tube. It is usually a non-linear piecewise continuous function. The dynamical behavior of such maps can be approximated by piecewise linear Markov maps (Boyarsky and Gora, 1997 Section 10.3). It is convenient to work with piecewise linear Markov maps on $I$ because the Frobenius-Perron operator of such maps are non-negative matrices with consecutive non-zero entries in each row and whose left eigenvectors, when viewed as functions, are the pdf values of the chaotic dynamical systems defined by $\tau$. For a given Markov map $\tau$ on a partition $\mathscr{P}$ of the state space, we refer to the associated matrix obtained by replacing all the non-zero entries by 1 as the transition matrix associated with $\tau$.

A dynamical system defined by a map $\tau$ and its pdf $f$ is chaotic if it is mixing (Boyarsky and Gora 1997, Ch. 3). If $\tau$ is piecewise linear and Markov, mixing can be defined in terms of an easily verifiable matrix property: $\tau$ is mixing if and only if the associated transition matrix $M$ is primitive, that is, there exists an integer $m$ such that all the entries of $M^{m}$ are positive. If a transition matrix does not have this property, then the associated dynamical system defined by $\tau$ is not chaotic and behaves in a completely predictable periodic manner.

DEFinition 3 (matrix definition of irreducible complexity). Let $\tau$ be a piecewise linear Markov map on the state space $I$ with respect to the partition, $\mathscr{P}$, consisting of $n$ disjoint subintervals of $I$. We let $M$ denote the associated matrix representation of the Frobenius-Perron operator. We say $\tau$ is irreducibly complex if $M$ is primitive but no principal submatrix (obtained by deleting the $i$ th row and $i$ th column, $i=1, \ldots, n)$ is primitive.

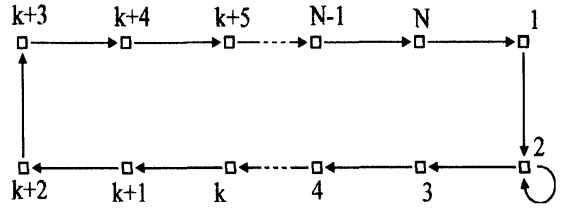

FIGURE 2

Example 1: Let the piecewise linear Markov transformation $\tau$ be defined on the 14-element equal partition $\mathscr{P}=\left\{I_{1}, I_{2}, \ldots, I_{13}, I_{14}\right\}$ of the interval $[0,1]$. We set $\tau\left(I_{1}\right)=I_{2}, \quad \tau\left(I_{2}\right)=I_{2} \cup I_{3}, \quad \tau\left(I_{3}\right)=I_{4}, \ldots, \tau\left(I_{13}\right)=I_{14}$, $\tau\left(I_{14}\right)=I_{1}$, as in Fig. 1 .

The transformation is defined by:

$$
\tau(x)=\left\{\begin{array}{cc}
\frac{2}{14}-x & x \leq \frac{1}{14} \\
\frac{1}{14}+2\left(x-\frac{1}{14}\right) & \frac{1}{14}<x \leq \frac{2}{14} \\
x+\frac{1}{14} & \frac{2}{14}<x \leq \frac{13}{14} \\
x-\frac{13}{14} & \frac{13}{14}<x \leq 1
\end{array}\right.
$$

The $0-1$ transition matrix associated with $\tau$ is given by:

$$
M=\left(\begin{array}{cccccccccccccc}
0 & 1 & 0 & 0 & 0 & 0 & 0 & 0 & 0 & 0 & 0 & 0 & 0 & 0 \\
0 & 1 & 1 & 0 & 0 & 0 & 0 & 0 & 0 & 0 & 0 & 0 & 0 & 0 \\
0 & 0 & 0 & 1 & 0 & 0 & 0 & 0 & 0 & 0 & 0 & 0 & 0 & 0 \\
0 & 0 & 0 & 0 & 1 & 0 & 0 & 0 & 0 & 0 & 0 & 0 & 0 & 0 \\
& & & & & & \ldots & & & & & & \\
0 & 0 & 0 & 0 & 0 & 0 & 0 & 0 & 0 & 0 & 0 & 0 & 1 & 0 \\
0 & 0 & 0 & 0 & 0 & 0 & 0 & 0 & 0 & 0 & 0 & 0 & 0 & 1 \\
1 & 0 & 0 & 0 & 0 & 0 & 0 & 0 & 0 & 0 & 0 & 0 & 0 & 0
\end{array}\right) .
$$

If the intervals are considered to be nodes of a graph, we have communication (Berman and Plemmons, 1979) between any two subintervals and there is at least one diagonal element equal to 1 , so this matrix is primitive, i.e. some power $M^{k}$ is positive. If, for any $i=1,2, \ldots, 14$, we remove the $i$ th column and $i$ th row of the matrix $M$, the resulting matrix $M_{i}$ is no longer primitive. Then, it has an attracting state. Thus, for the dynamical system defined by $\tau$, removal of any interval of states, causes a collapse to fixed periodic behavior. The condition of Definition 2 is satisfied for $\varepsilon=2 / 14$. Then any interval of this length will completely contain one of the partition subintervals. A similar construction can be done for any $N \geq 3$.

Example 2: Piecewise linear Markov maps with $N$ branches possessing the irreducible complexity property.

We construct $\tau$ to realize the $N$-node graph in Fig. 2. It is enough to show that the graph is primitive when complete and that it collapses into a periodic or a "one attracting node" graph after removal of any particular node. The complete graph is primitive since there is communication between any two nodes and node 2 is self-communicating, which corresponds to a 1 on the diagonal of the associated 0-1 matrix. If any other node is removed, the graph 


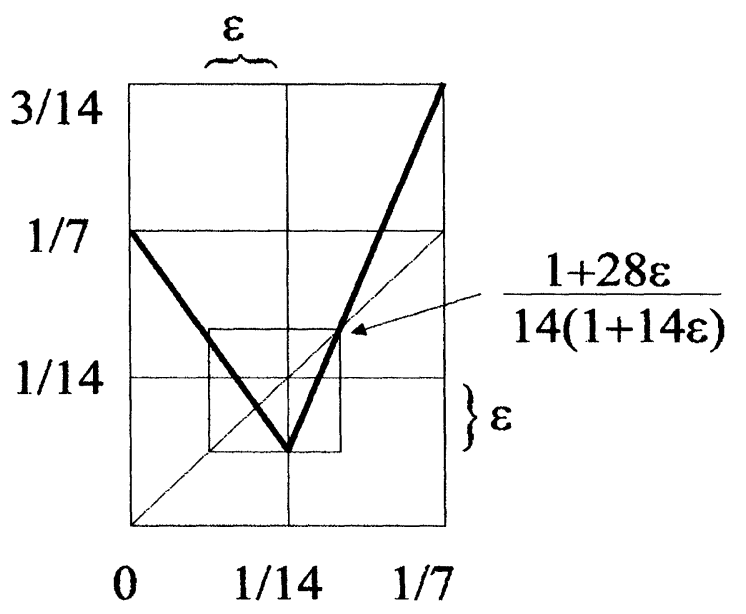

FIGURE 3

becomes an absorbing graph: if node $s$ is removed, then node $s-1$ becomes a global attractor.

Note that there are two phenomena apparent in Fig. 2. One is a folding effect which is essential for chaos: node $2 \rightarrow$ node 2 and node $2 \rightarrow$ node 3 . This is the chaos engine of the dynamical system. Then there is the cascading effect as evinced by the direct flow from one node to the other throughout the graph. Cascading is an important process in organisms (Boyarsky and Gora, 1997).

\section{DISCONTINUOUS DYNAMICAL BEHAVIOR}

Let us consider once again the map $\tau$ of Example 1. Now, instead of removing an interval from the state space, we perturb the map slightly, obtaining $\tau_{\varepsilon}$. From the matrix representation of the Frobenius-Perron operator it is easy to show that invariant measure is equivalent to the Lebesgue measure $\lambda$. More precisely, it is $\mu=f \cdot \lambda$, where

$$
f=\frac{14}{15}\left(\chi_{I_{1}}+2 \chi_{I_{2}}+\chi_{I_{3}}+\ldots+\chi_{I_{14}}\right)
$$

Now, for any $1 / 14>\varepsilon>0$, we shall construct a map $\tau_{\varepsilon}$, which is $\varepsilon$ close to $\tau$ yet its absolutely continuous invariant measure is very different from measure $\mu$ and the Lebesgue measure.

Let us change $\tau$ on intervals $I_{1}$ and $I_{2}$, as shown in Fig. 3 . Let

$$
\tau_{\varepsilon}(x)=\left\{\begin{array}{cc}
\frac{2}{14}-(1+14 \varepsilon) x, & x \leq \frac{1}{14} \\
\frac{1}{14}-\varepsilon+(2+14 \varepsilon)\left(x-\frac{1}{14}\right), & \frac{1}{14}<x \leq \frac{2}{14}
\end{array}\right.
$$

and

$$
\tau_{\varepsilon}(x)=\tau(x) \quad \text { on } \quad\left[\frac{2}{14}, 1\right]
$$

The interval $I_{\varepsilon}=[(1 / 14)-\varepsilon,(1+28 \varepsilon) /(14(1+$ $14 \varepsilon))]$ is $\tau_{\varepsilon}$ invariant. Since the transformation $\tau_{\varepsilon}$ is still mixing, i.e. there is communication between any two subintervals $I_{1}, I_{2}, \ldots, I_{14}$, the absolutely continuous $\tau_{\varepsilon^{-}}$ invariant measure $\mu_{\varepsilon}$ is concentrated on the interval $I_{\varepsilon}$. As $\varepsilon \rightarrow 0$, the maps $\tau_{\varepsilon}$ converge to $\tau$, but the measures $\mu_{\varepsilon}$ converge to the $\delta$-measure at $1 / 14$ and not to the measure $\mu$. Again, this example can be generalized for any $N \geq 3$. The idea behind this example is inspired by an example of Keller (1982).

Since a system cannot jump abruptly from being totally non-functioning to a fully functioning system, this type of example argues against selective evolution as a general means of achieving the dynamical behavior of a complex system by means of small perturbations.

\section{Acknowledgements}

This research has been supported by NSERC grants.

\section{References}

Behe, M.J. (1996) Darwin's Black Box (Touchstone).

Berman, A. and Plemmons, R. (1979) Nonnegative Matrices in the Mathematical Sciences (Academic Press, New York).

Boyarsky, A. and Góra, P. (1997) Laws of Chaos (Birkhauser, Basel).

Caplan, S.R. and Kara-Ivanov, M. (1993) "The bacterial flagellar motor", Int. Rev. Cytology 147, 97-164.

Guevera, M.R. and Glass, L. (1982) "Phase-locking, period doubling, bifurcations and chaos in a mathematical model of a periodically driven oscillator: A theory for the entrainment of biological oscillators and the generation of cardiac dysrhythmia", J. Math. Biol. 14, 1-23.

Keller, G. (1982) "Stochastic stability in some chaotic dynamical systems", Mh. Math. 94, 313-333.

Lasota, A. and Rusck, P. (1974) "An application of ergodic theory to the determination of the efficiency of cogged drilling bits", Arch. Gornictwa 19, 281-295.

Liebovitch, L.S. and Krekora, P. (2000) "The physical basis of ion channel kinetics; the importance of dynamics", Proc. Inst. Math. Appl. (IMA).

May, R. (1976) "Simple mathematical models with very complicated dynamics", Nature 261, 459-467.

Schuster, S.C. and Kahn, S. (1994) "The bacterial flagellar motor", Ann. Rev. Biophy. Biomol. Struct. 23, 509-539.

Shinbrot, T., Grebogi, C., Wisdom, J. and Yorke, J.A. (1992) "Chaos in a double pendulum", Am. J. Phys. 60(6), 491-499.

West, B.J. (1990) Fractal Physiology and Chaos in Medicine (World Scientific, Singapore).

Yuan, G.-C., Yorke, J.A., Carroll, T.L., Ott, E. and Pecora, L.M. (2000) "Testing whether two chaotic one-dimensional processes are dynamically identical", Phys. Rev. Lett. 85(20), 4265-4268. 


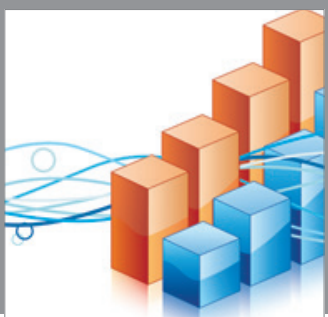

Advances in

Operations Research

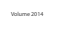

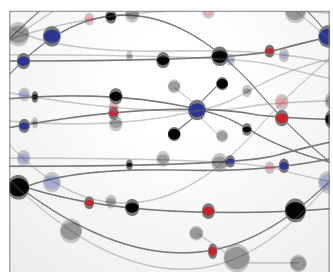

\section{The Scientific} World Journal
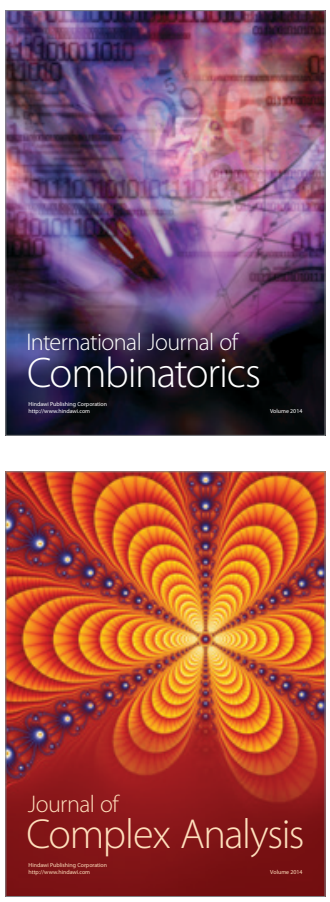

International Journal of

Mathematics and

Mathematical

Sciences
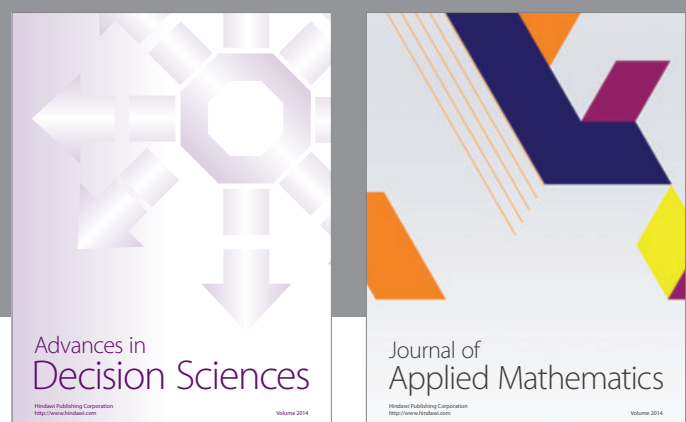

Journal of

Applied Mathematics
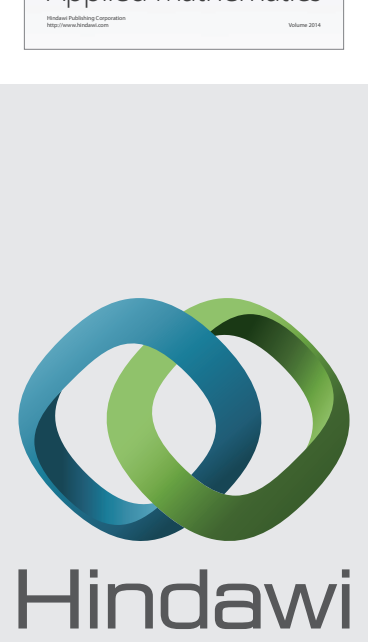

Submit your manuscripts at http://www.hindawi.com
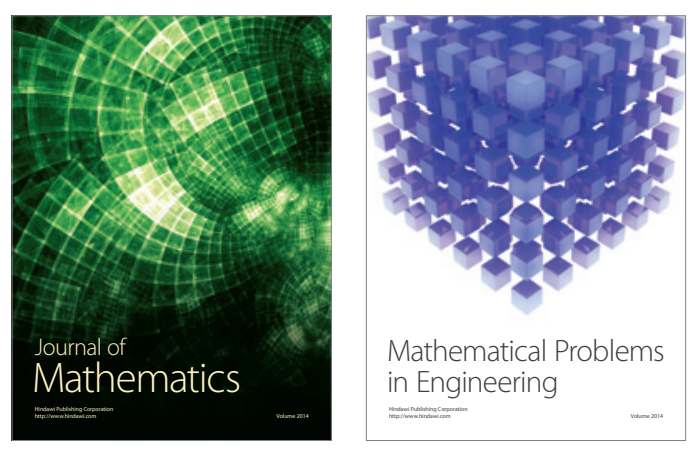

Mathematical Problems in Engineering
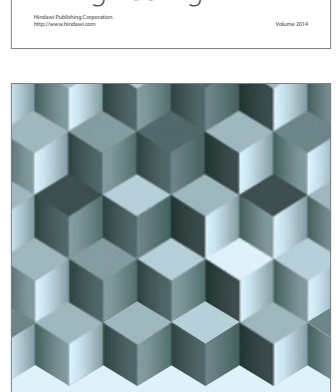

Journal of

Function Spaces
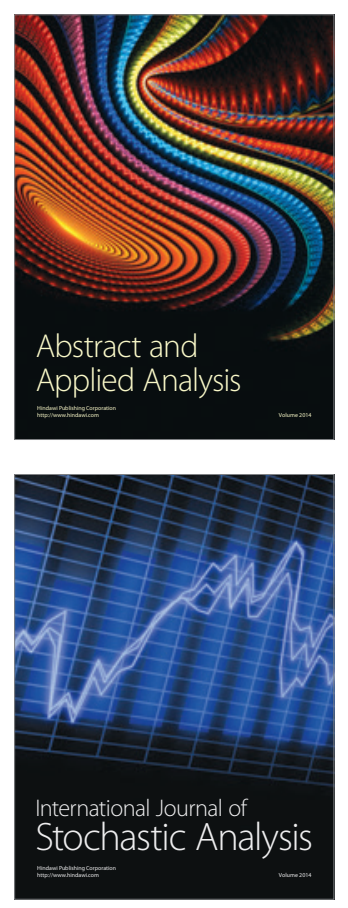

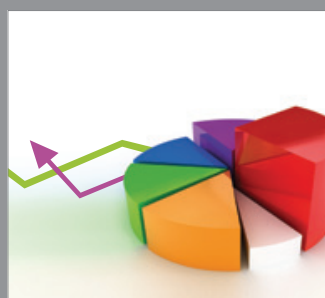

ournal of

Probability and Statistics

Promensencen
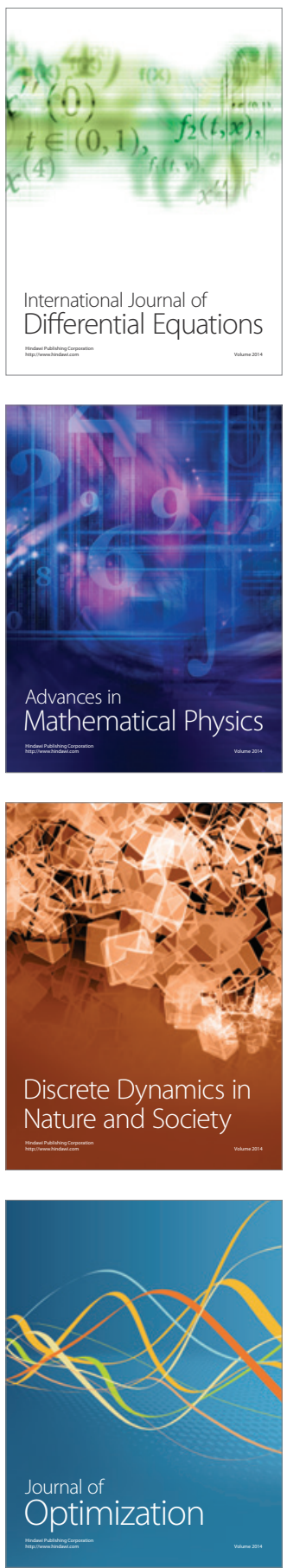